\title{
Treatment Patterns for Gastroesophageal Junction Adenocarcinoma in the United States ${ }^{\dagger}$
}

\author{
Bradford J. Kim ${ }^{1}$ (D, Yi-Ju Chiang ${ }^{1}$, Prajnan Das ${ }^{2}$, Bruce D. Minsky ${ }^{2}$, Mariela A. Blum ${ }^{3}$, \\ Jaffer A. Ajani ${ }^{3}$, Jeannelyn S. Estrella ${ }^{4}$, Wayne L. Hofstetter ${ }^{5}$, Ching-Wei D. Tzeng ${ }^{1}$, \\ Brian D. Badgwell ${ }^{1}$, Paul F. Mansfield ${ }^{1}$ and Naruhiko Ikoma ${ }^{1, *}$
}

1 Department of Surgical Oncology, The University of Texas MD Anderson Cancer Center, 1400 Pressler Drive, Unit 1484, Houston, TX 77030, USA; bjkim@mdanderson.org (B.J.K.); YChiang1@mdanderson.org (Y.-J.C.); CDTzeng@mdanderson.org (C.-W.D.T.); bbadgwell@mdanderson.org (B.D.B.); pmansfie@mdanderson.org (P.F.M.)

2 Department of Radiation Oncology, The University of Texas MD Anderson Cancer Center, 1400 Pressler Drive, Unit 1484, Houston, TX 77030, USA; PrajDas@mdanderson.org (P.D.); BMinsky@mdanderson.org (B.D.M.)

3 Department of Gastrointestinal Medical Oncology, The University of Texas MD Anderson Cancer Center, 1400 Pressler Drive, Unit 1484, Houston, TX 77030, USA; mblum@mdanderson.org (M.A.B.); jajani@mdanderson.org (J.A.A.)

4 Department of Pathology, The University of Texas MD Anderson Cancer Center, 1400 Pressler Drive, Unit 1484, Houston, TX 77030, USA; JSEstrella@mdanderson.org

5 Department of Thoracic and Cardiovascular Surgery, The University of Texas MD Anderson Cancer Center, 1400 Pressler Drive, Unit 1484, Houston, TX 77030, USA; WHofstetter@mdanderson.org

* Correspondence: NIkoma@mdanderson.org; Tel.: +1-713-563-0766; Fax: +1-713-794-5720

+ Presentation of Data: These data were presented at the 120th Annual Japan Surgical Society Congress, 13-15 August 2020 and Annual Meeting for Society of Surgical Oncology, 17-18 August 2020.

Received: 6 October 2020; Accepted: 15 October 2020; Published: 29 October 2020

\begin{abstract}
Despite the increasing incidence of gastroesophageal junction adenocarcinoma (GEJA), the optimal treatment strategy for the disease remains unknown. The objective of this study was to describe treatment patterns for GEJA in the United States. The National Cancer Database was searched to identify all patients who underwent resection of the lower esophagus, abdominal esophagus, and/or gastric cardia for GEJA between 2006 and 2016. Patients were grouped by clinical disease stage: early localized (L; T1-2N0), locally advanced (LA; T3-4N0), regional (R; T1-2N+), or regionally advanced (RA; T3-4N+). The search identified 28,852 GEJA patients. The dominant age range was 60-69 years (39\%). Most patients were men (85\%), and most were white $(92 \%)$. Most L patients $(69 \%)$ underwent upfront surgery, whereas most LA, R, and RA patients received neoadjuvant therapy (NAT; $86 \%, 80 \%$, and 90\%, respectively). Among patients who received NAT, $85 \%$ received chemoradiotherapy. Adjuvant therapy was relatively uncommon across all groups (15-20\%). In the LA, R, and RA groups, overall survival was greater in patients who received NAT compared to upfront surgery $(p<0.001)$. With the exception of patients with early localized node-negative disease, most GEJA patients receive neoadjuvant chemoradiotherapy despite the lack of prospective trials reporting survival benefit over chemotherapy alone.
\end{abstract}

Keywords: chemoradiation; esophagectomy; gastrectomy; GEJ; neoadjuvant therapy

\section{Introduction}

In the early 1970s, the incidence of gastroesophageal junction adenocarcinoma (GEJA) began rising, increasing by nearly 2.5 -fold into the early 1990 s, when it stabilized into a plateaued rate [1]. 
During this time, the advent of the Siewert classification was used to improve treatment plans of adenocarcinomas localized to the gastroesophageal junction (GEJ) [2,3]. Broadly, Siewert defined a GEJ tumor as one with its center within $5 \mathrm{~cm}$ proximal and distal to the anatomic gastric cardia [3].

From the past two decades, several large randomized studies have demonstrated significant survival benefit from pre- and/or postoperative therapies in the treatment of gastric [4-7] and esophageal $[8,9]$ cancer. However, no phase III trial has studied adenocarcinoma localized only to the GEJ independently, and treatment recommendations are based on studies mostly focused on gastric or esophageal cancers [10]. In brief, the phase III Medical Research Council Adjuvant Gastric Infusional Chemotherapy (MAGIC) trial, which included gastric and GEJ cancers, showed the survival benefit of perioperative chemotherapy over surgery alone. The phase III Chemoradiotherapy for Oesophageal Cancer Followed by Surgery Study (CROSS) trial, which included esophageal and GEJ cancers, showed the survival benefit of preoperative chemoradiation therapy over surgery alone. Based on these landmark trials and subsequent prospective studies that reproduced the benefit of such treatment approaches, both preoperative chemotherapy and chemoradiation therapy are recommended in treatment guidelines for GEJA [10]. Currently, there is a paucity of data that specifically investigate the pre- and/or postoperative treatment approaches for GEJA [11,12], and the optimal treatment remains unknown. To address this gap in knowledge, the objective of this study was to describe current treatment patterns for patients who underwent resection for GEJA in this era of increased incidence and use of modern treatment modalities. We therefore examined the current practice pattern in the United States for patients with resectable GEJA by using the largest nationwide cancer database available.

\section{Methods}

\subsection{Dataset}

The National Cancer Database (NCDB) was used for this retrospective cohort analysis. Jointly maintained by the American Cancer Society and American College of Surgeons Commission on Cancer, the NCDB is a prospectively collected hospital-based cancer registry of more than 1500 Commission on Cancer-accredited centers in the United States. This study was approved by the Institutional Review Board of The University of Texas MD Anderson Cancer Center.

\section{Patient Selection}

The NCDB was searched to identify all patients who underwent resection of the lower esophagus, abdominal esophagus, and/or gastric cardia for GEJA between 2006 and 2016. First, the NCDB was queried for all patients 18 years and older with International Classification of Disease of Oncology, Third Edition (ICD-O-3) topography codes C152-C155 (C152, abdominal esophagus; C153, upper third of the esophagus; C154, middle third of the esophagus; $\mathrm{C} 155$, lower third of the esophagus) and C160-C166 (gastric cardia, gastric fundus, gastric body, gastric antrum, gastric pylorus, gastric lesser curvature, and gastric greater curvature). Target histology included: 8140 (adenocarcinoma, not otherwise specified), 8144 (adenocarcinoma, intestinal type), 8145 (adenocarcinoma, diffuse type), and 8490 (signet ring cell adenocarcinoma). All patients who underwent gastrectomy or esophagectomy were identified, and patients without neoadjuvant/adjuvant therapy records were excluded from the analysis. Patients with GEJA were defined as patients who had adenocarcinoma of the abdominal esophagus, lower third of the esophagus, or gastric cardia.

\subsection{Clinical Variables}

Patient demographics including race, gender, age, high school education (\% who did not graduate), zip code income, and insurance status were collected. The Charlson Comorbidity Index was included in the analysis. Cancer-related variables included clinical and pathologic TNM (tumor, nodes, and metastases) cancer stage, margin status (R0, R1, R2, or RX), tumor size measured at the time of pathology exam, receipt of lymphadenectomy, adequate lymph node sampling, receipt of neoadjuvant 
chemotherapy and/or adjuvant chemotherapy, receipt of neoadjuvant and/or adjuvant radiation therapy, mortality, and overall survival. Adequate lymph node sampling was defined as a retrieval of 15 or more lymph nodes $(\mathrm{LN} \geq 15)$ per AJCC guidelines. Neoadjuvant therapy (NAT) was defined as any treatment before surgery, including any combination of chemotherapy and/or radiation therapy. Similarly, adjuvant therapy was defined as any therapy received after surgical resection. Patients with any lymph nodes positive for adenocarcinoma were considered node positive $(\mathrm{N}+)$. Patients were grouped by clinical disease stage: early localized (L; T1-2N0), locally advanced (LA; T3-4N0), regional (R; T1-2N+), or regionally advanced (RA; T3-4N+), and practice patterns and treatment outcomes were stratified by these stage groups.

\subsection{Statistical Analysis}

The yearly overall reported number of GEJA cases was calculated as a subset of all yearly adenocarcinoma patients, and the yearly number of resected GEJA was calculated as a subset of all yearly GEJA patients. The Mann-Whitney U-test was used for comparison of continuous data. Categorical data were compared with the Chi-squared test or Fisher's exact test. Kaplan-Meier estimates and log-rank tests were used to calculate and compare the survival curves, median survival time, and the five-year overall survival for treatment by clinical stage. Multivariable analysis for overall survival in all patients was conducted to investigate factors, including use of NAT, associated with overall survival (OS). A logistic regression model was used to assess the likelihood of NAT vs. surgery first. Cox regression models were used to determine predictors of survival. Interaction terms were evaluated for different survival impacts of NAT by clinical stage to determine appropriate Cox regression models. Covariates with $p<0.2$ from univariate analysis were retained, then a stepwise selection method was performed to determine the final logistic and Cox regression models with relevant variables. The Hosmer-Lemeshow goodness-of-fit test was applied to test the final model. Odds ratios (OR), hazard ratios (HR), and 95\% confidence intervals ( $95 \% \mathrm{CI}$ ) were estimated for the NAT and survival outcomes. All tests were two-sided. A $p$ value $<0.05$ was the significance level for all analyses. All data analyses were conducted using SAS Enterprise Guide 7.15 (SAS Inc., Cary, NC, USA).

\section{Results}

Initial analysis of the NCDB identified 239,018 patients from a combined cohort of esophageal and gastric cancer patients. From this group, there were 203,195 patients with squamous cell carcinoma or adenocarcinoma, 72,382 of whom had undergone resection with a gastrectomy or esophagectomy. After excluding patients without a record of neoadjuvant/adjuvant therapy, there remained 71,058 patients who had undergone gastrectomy or esophagectomy for squamous cell carcinoma or adenocarcinoma of any location in these two organs. The distribution for tumor location is shown in Table 1. The majority of patients in this cohort had tumor histology of adenocarcinoma $(n=214,281 ; 83.8 \%)$, and the distribution of adenocarcinoma is shown in Table A1.

The total cohort of this study for further analyses included 28,852 GEJA patients, with most patients being male (85.3\%) and white (92.1\%). This analysis identified 8286 (28.7\%) L, 5678 (19.7\%) LA, $3327(11.5 \%)$ R, and 11,561 (40.1\%) RA GEJA patients. Demographic, clinical, and treatment pattern variables are listed in Table 2. Most patients with clinical stage L GEJA (5710, 68.9\%) underwent upfront surgery, whereas most patients with LA $(4918,86.6 \%)$, R (2659, 79.9\%), and RA $(10,512,90.9 \%)$ GEJA received NAT.

The use of NAT constantly increased over the study period in all clinical stages and is represented in Figure $1(p<0.001)$. Among patients who received NAT, 85\% received chemoradiation, $13 \%$ chemotherapy alone, and $2 \%$ radiation therapy alone. Neoadjuvant chemoradiation was the most common modality among patients who received NAT across all clinical stages (L: $25.5 \%$, LA: $75.2 \%$, R: 68.1, RA: $78.0 \% ; p<0.001)$. The use of adjuvant therapy was relatively uncommon (16.4\%) across all groups (L: $14.1 \%$ LA: $14.8 \%$ R: $18.2 \%$ RA: $18.3 \%, p<0.001)$. Among patients with 
clinical stage L and LA disease, 1788 (21.6\%) and 1717 (30.2\%), respectively, were pathologically node positive after surgery. Among patients with clinical N-negative but pathologic N-positive disease, most patients with clinical stage $\mathrm{L}$ disease underwent upfront resection $(1229,67.9 \%)$ rather than neoadjuvant chemotherapy $(18,1.0 \%)$, neoadjuvant radiation $(128,7.1 \%)$, and neoadjuvant chemoradiation $(435,24.0 \%)$. Most patients with clinical stage LA disease underwent neoadjuvant chemoradiation $(1149,65.4 \%)$ before resection. Lastly, among patients with clinical stage LA disease, a greater proportion of surgery-first patients $(361,47.5 \%)$ were upstaged to pathological $\mathrm{N}+$ disease compared to those who received NAT $(1397,28.4 \% ; p<0.001)$. Multivariable logistic regression analyses to identify factors associated with receiving NAT vs. surgery first are represented in Table 3. The use of NAT was associated with esophageal classification (OR 1.57), male gender (OR 1.33), white race (OR 1.27), younger age (OR 1.52; age $<50$, when compared to age 70-79), and lower Charlson Comorbidity Index (OR 1.43; score 0 , when compared to score $\geq 2$ ), and advanced clinical stage (OR 2.53; T3-T4N+, when compared to T1-2N+).

Table 1. Yearly number of all tumors of the esophagus and stomach and number resected by its anatomic location.

\begin{tabular}{|c|c|c|c|c|c|c|c|c|c|c|c|}
\hline \multirow[t]{2}{*}{ Esophagus } & \multirow{2}{*}{$\begin{array}{c}\text { Overall } \\
n\end{array}$} & \multicolumn{2}{|c|}{$\begin{array}{c}\text { Overall } \\
\text { Resected }\end{array}$} & \multicolumn{2}{|c|}{ Upper } & \multicolumn{2}{|c|}{ Middle } & \multicolumn{2}{|c|}{ Lower } & \multicolumn{2}{|c|}{ Abdominal } \\
\hline & & $n$ & $\%$ & $n$ & $\%$ & $n$ & $\%$ & $n$ & $\%$ & $n$ & $\%$ \\
\hline 2006 & 9591 & 2637 & 27.5 & 54 & 2.0 & 245 & 9.3 & 1952 & 74.0 & 22 & 0.8 \\
\hline 2007 & 9817 & 2650 & 27.0 & 46 & 1.7 & 229 & 8.6 & 2004 & 75.6 & 21 & 0.8 \\
\hline 2008 & 10,061 & 2654 & 26.4 & 40 & 1.5 & 215 & 8.1 & 2004 & 75.5 & 22 & 0.8 \\
\hline 2009 & 10,308 & 2615 & 25.4 & 37 & 1.4 & 206 & 7.9 & 1987 & 76.0 & 11 & 0.4 \\
\hline 2010 & 10,033 & 2455 & 24.5 & 37 & 1.5 & 226 & 9.2 & 1822 & 74.2 & 14 & 0.6 \\
\hline 2011 & 10,205 & 2531 & 24.8 & 35 & 1.4 & 255 & 10.1 & 1902 & 75.1 & 14 & 0.6 \\
\hline 2012 & 10,578 & 2500 & 23.6 & 38 & 1.5 & 238 & 9.5 & 1903 & 76.1 & 9 & 0.4 \\
\hline 2013 & 10,999 & 2722 & 24.7 & 42 & 1.5 & 262 & 9.6 & 2088 & 76.7 & 5 & 0.2 \\
\hline 2014 & 11,095 & 2648 & 23.9 & 33 & 1.2 & 251 & 9.5 & 2039 & 77.0 & 10 & 0.4 \\
\hline 2015 & 11,524 & 2651 & 23.0 & 40 & 1.5 & 204 & 7.7 & 2101 & 79.3 & 7 & 0.3 \\
\hline 2016 & 11,471 & 2627 & 22.9 & 33 & 1.3 & 235 & 8.9 & 2061 & 78.5 & 7 & 0.3 \\
\hline \multirow[t]{2}{*}{ Gastric } & Overall & \multicolumn{2}{|c|}{$\begin{array}{c}\text { Overall } \\
\text { Resected }\end{array}$} & \multicolumn{2}{|c|}{ Cardia } & \multicolumn{2}{|c|}{ Proximal } & \multicolumn{2}{|c|}{ Distal } & \multicolumn{2}{|c|}{$\begin{array}{c}\text { Lesser/Greater } \\
\text { Curvature }\end{array}$} \\
\hline & $n$ & $n$ & $\%$ & $n$ & $\%$ & $n$ & $\%$ & $n$ & $\%$ & $n$ & $\%$ \\
\hline 2006 & 10,955 & 5101 & 46.6 & 1610 & 31.6 & 549 & 10.8 & 1229 & 24.1 & 649 & 12.7 \\
\hline 2007 & 11,332 & 5146 & 45.4 & 1513 & 29.4 & 553 & 10.7 & 1291 & 25.1 & 711 & 13.8 \\
\hline 2008 & 11,485 & 5090 & 44.3 & 1473 & 28.9 & 579 & 11.4 & 1294 & 25.4 & 690 & 13.6 \\
\hline 2009 & 12,110 & 5225 & 43.1 & 1610 & 30.8 & 583 & 11.2 & 1354 & 25.9 & 655 & 12.5 \\
\hline 2010 & 12,532 & 5230 & 41.7 & 1758 & 33.6 & 561 & 10.7 & 1281 & 24.5 & 708 & 13.5 \\
\hline 2011 & 13,047 & 5410 & 41.5 & 1887 & 34.9 & 562 & 10.4 & 1337 & 24.7 & 732 & 13.5 \\
\hline 2012 & 13,317 & 5391 & 40.5 & 1814 & 33.6 & 520 & 9.6 & 1371 & 25.4 & 696 & 12.9 \\
\hline 2013 & 13,845 & 5460 & 39.4 & 1927 & 35.3 & 653 & 12.0 & 1401 & 25.7 & 636 & 11.6 \\
\hline 2014 & 13,964 & 5409 & 38.7 & 1924 & 35.6 & 697 & 12.9 & 1315 & 24.3 & 616 & 11.4 \\
\hline 2015 & 13,911 & 5226 & 37.6 & 1951 & 37.3 & 643 & 12.3 & 1232 & 23.6 & 604 & 11.6 \\
\hline 2016 & 13,622 & 4976 & 36.5 & 1817 & 36.5 & 615 & 12.4 & 1191 & 23.9 & 584 & 11.7 \\
\hline
\end{tabular}


Table 2. Demographic, clinical, and treatment variables for patients who underwent resection of gastroesophageal junction adenocarcinoma tumors by clinical stage.

\begin{tabular}{|c|c|c|c|c|c|c|c|c|c|c|c|}
\hline & \multicolumn{2}{|c|}{ Total } & \multicolumn{2}{|c|}{ T1/T2 N0 } & \multicolumn{2}{|c|}{ T3/T4 N0 } & \multicolumn{2}{|c|}{ T1/T2 N+ } & \multicolumn{2}{|c|}{ T3/T4 N+ } & \multirow{2}{*}{$p$} \\
\hline & $n=28,852$ & $\%$ & $n=8286$ & $\%$ & $n=5678$ & $\%$ & $n=3327$ & $\%$ & $n=11,561$ & $\%$ & \\
\hline Gender & & & & & & & & & & & $<0.001$ \\
\hline Male & 24,611 & 85.3 & 6900 & 83.3 & 4818 & 84.9 & 2878 & 86.5 & 10,015 & 86.6 & \\
\hline Age & & & & & & & & & & & $<0.001$ \\
\hline$<50$ & 2622 & 9.1 & 581 & 7.0 & 508 & 9.0 & 303 & 9.1 & 1230 & 10.6 & \\
\hline $50-59$ & 7128 & 24.7 & 1809 & 21.8 & 1308 & 23.0 & 826 & 24.8 & 3185 & 27.6 & \\
\hline $60-69$ & 11,144 & 38.6 & 3102 & 37.4 & 2219 & 39.1 & 1344 & 40.4 & 4479 & 38.7 & \\
\hline $70-79$ & 6862 & 23.8 & 2324 & 28.1 & 1414 & 24.9 & 746 & 22.4 & 2378 & 20.6 & \\
\hline$\geq 80$ & 1096 & 3.8 & 470 & 5.7 & 229 & 4.0 & 108 & 3.3 & 289 & 2.5 & \\
\hline Race & & & & & & & & & & & 0.758 \\
\hline White & 26,572 & 92.1 & 7607 & 91.8 & 5251 & 92.5 & 3057 & 91.9 & 10,657 & 92.2 & \\
\hline Black & 690 & 2.4 & 211 & 2.6 & 126 & 2.2 & 79 & 2.4 & 274 & 2.4 & \\
\hline Hispanic & 836 & 2.9 & 232 & 2.8 & 156 & 2.8 & 105 & 3.2 & 343 & 3.0 & \\
\hline Asian/Pacific Islander & 372 & 1.3 & 118 & 1.4 & 73 & 1.3 & 34 & 1.0 & 147 & 1.3 & \\
\hline Other & 161 & 0.6 & 50 & 0.6 & 30 & 0.5 & 25 & 0.8 & 56 & 0.5 & \\
\hline Unknown & 221 & 0.8 & 68 & 0.8 & 42 & 0.7 & 27 & 0.8 & 84 & 0.7 & \\
\hline Anatomical Location & & & & & & & & & & & $<0.001$ \\
\hline Abdominal esophagus & 80 & 0.3 & 27 & 0.3 & 9 & 0.2 & 10 & 0.3 & 34 & 0.3 & \\
\hline Lower esophagus & 15,380 & 53.3 & 4239 & 51.2 & 3006 & 52.9 & 1833 & 55.1 & 6302 & 54.5 & \\
\hline Gastric cardia & 13,392 & 46.4 & 4020 & 48.5 & 2663 & 46.9 & 1484 & 44.6 & 5225 & 45.2 & \\
\hline Organ & & & & & & & & & & & $<0.001$ \\
\hline Esophagus & 15,460 & 53.6 & 4266 & 51.5 & 3015 & 53.1 & 1843 & 55.4 & 6336 & 54.8 & \\
\hline Stomach & 13,392 & 46.4 & 4020 & 48.5 & 2663 & 46.9 & 1484 & 44.6 & 5225 & 45.2 & \\
\hline Facility Type & & & & & & & & & & & $<0.001$ \\
\hline Community Program & 9619 & 33.3 & 2663 & 32.1 & 2120 & 37.3 & 1089 & 32.7 & 3747 & 32.4 & \\
\hline Academic Program & 19,233 & 66.7 & 5623 & 67.9 & 3558 & 62.7 & 2238 & 67.3 & 7814 & 67.6 & \\
\hline \multicolumn{12}{|l|}{ Comorbidity Score } \\
\hline 0 & 20,113 & 69.7 & 5431 & 65.5 & 3999 & 70.4 & 2355 & 70.8 & 8328 & 72.0 & \\
\hline 1 & 6566 & 22.8 & 2084 & 25.2 & 1274 & 22.4 & 741 & 22.3 & 2467 & 21.3 & \\
\hline$\geq 2$ & 2173 & 7.5 & 771 & 9.3 & 405 & 7.1 & 231 & 6.9 & 766 & 6.6 & \\
\hline
\end{tabular}


Table 2. Cont.

\begin{tabular}{|c|c|c|c|c|c|c|c|c|c|c|c|}
\hline & \multicolumn{2}{|c|}{ Total } & \multicolumn{2}{|c|}{ T1/T2 N0 } & \multicolumn{2}{|c|}{ T3/T4 N0 } & \multicolumn{2}{|c|}{$\mathrm{T} 1 / \mathrm{T} 2 \mathrm{~N}+$} & \multicolumn{2}{|c|}{ T3/T4 N+ } & \multirow{2}{*}{$p$} \\
\hline & $n=28,852$ & $\%$ & $n=8286$ & $\%$ & $n=5678$ & $\%$ & $n=3327$ & $\%$ & $n=11,561$ & $\%$ & \\
\hline Education & & & & & & & & & & & 0.017 \\
\hline$\geq 29 \%$ & 3202 & 11.1 & 909 & 11.0 & 694 & 12.2 & 378 & 11.4 & 1221 & 10.6 & \\
\hline$<29 \%$ & 24,855 & 86.2 & 7152 & 86.3 & 4810 & 84.7 & 2852 & 85.7 & 10,041 & 86.9 & \\
\hline Unknown & 795 & 2.8 & 225 & 2.7 & 174 & 3.1 & 97 & 2.9 & 299 & 2.6 & \\
\hline Zip Code Income & & & & & & & & & & & 0.184 \\
\hline$\geq \$ 46,000$ & 12,180 & 42.2 & 3461 & 41.8 & 2341 & 41.2 & 1400 & 42.1 & 4975 & 43.0 & \\
\hline$<\$ 46,000$ & 15,879 & 55.0 & 4598 & 55.5 & 3163 & 55.7 & 1830 & 55.0 & 6288 & 54.4 & \\
\hline Unknown & 793 & 2.8 & 224 & 2.7 & 174 & 3.1 & 97 & 2.9 & 298 & 2.6 & \\
\hline Insurance & & & & & & & & & & & $<0.001$ \\
\hline Private & 13,387 & 46.4 & 3378 & 40.8 & 2486 & 43.8 & 1645 & 49.4 & 5878 & 50.8 & \\
\hline Not Insured & 504 & 1.8 & 133 & 1.6 & 98 & 1.7 & 47 & 1.4 & 226 & 2.0 & \\
\hline Government's Plan & 14,520 & 50.3 & 4616 & 55.7 & 3006 & 52.9 & 1603 & 48.2 & 5295 & 45.8 & \\
\hline Unknown & 441 & 1.5 & 159 & 1.9 & 88 & 1.6 & 32 & 1.0 & 162 & 1.4 & \\
\hline Margin & & & & & & & & & & & $<0.001$ \\
\hline R0 & 26,114 & 90.5 & 7702 & 93.0 & 5079 & 89.5 & 3035 & 91.2 & 10,298 & 89.1 & \\
\hline $\mathrm{R} 1$ & 1101 & 3.8 & 207 & 2.5 & 253 & 4.5 & 111 & 3.3 & 530 & 4.6 & \\
\hline $\mathrm{R} 2$ & 59 & 0.2 & 11 & 0.1 & 12 & 0.2 & 6 & 0.2 & 30 & 0.3 & \\
\hline $\mathrm{RX}$ & 1578 & 5.5 & 366 & 4.4 & 334 & 5.9 & 175 & 5.3 & 703 & 6.1 & \\
\hline Pathologic T & & & & & & & & & & & $<0.001$ \\
\hline T0 & 3533 & 12.3 & 546 & 6.6 & 710 & 12.5 & 535 & 16.1 & 1742 & 15.1 & \\
\hline $\mathrm{T} 1$ & 6536 & 22.7 & 3961 & 47.8 & 644 & 11.3 & 822 & 24.7 & 1109 & 9.6 & \\
\hline $\mathrm{T} 2$ & 4217 & 14.6 & 1249 & 15.1 & 776 & 13.7 & 642 & 19.3 & 1550 & 13.4 & \\
\hline $\mathrm{T} 3$ & 8577 & 29.7 & 1034 & 12.5 & 2344 & 41.3 & 579 & 17.4 & 4620 & 40.0 & \\
\hline $\mathrm{T} 4$ & 462 & 1.6 & 53 & 0.6 & 148 & 2.6 & 32 & 1.0 & 229 & 2.0 & \\
\hline $\mathrm{TX}$ & 5527 & 19.2 & 1443 & 17.4 & 1056 & 18.6 & 717 & 21.6 & 2311 & 20.0 & \\
\hline Tumor Size & & & & & & & & & & & $<0.001$ \\
\hline$\leq 5 \mathrm{~cm}$ & 15,334 & 53.2 & 5586 & 67.4 & 2813 & 49.5 & 1796 & 54.0 & 5139 & 44.5 & \\
\hline$>5-10 \mathrm{~cm}$ & 4182 & 14.5 & 572 & 6.9 & 839 & 14.8 & 433 & 13.0 & 2338 & 20.2 & \\
\hline$>10-15 \mathrm{~cm}$ & 300 & 1.0 & 34 & 0.4 & 54 & 1.0 & 26 & 0.8 & 186 & 1.6 & \\
\hline$>15 \mathrm{~cm}$ & 222 & 0.8 & 91 & 1.1 & 42 & 0.7 & 25 & 0.8 & 64 & 0.6 & \\
\hline Unknown & 8814 & 30.6 & 2003 & 24.2 & 1930 & 34.0 & 1047 & 31.5 & 3834 & 33.2 & \\
\hline
\end{tabular}


Table 2. Cont.

\begin{tabular}{|c|c|c|c|c|c|c|c|c|c|c|c|}
\hline & \multicolumn{2}{|c|}{ Total } & \multicolumn{2}{|c|}{ T1/T2 N0 } & \multicolumn{2}{|c|}{ T3/T4 N0 } & \multicolumn{2}{|c|}{$\mathrm{T} 1 / \mathrm{T} 2 \mathrm{~N}+$} & \multicolumn{2}{|c|}{ T3/T4 N+ } & \multirow{2}{*}{$p$} \\
\hline & $n=28,852$ & $\%$ & $n=8286$ & $\%$ & $n=5678$ & $\%$ & $n=3327$ & $\%$ & $n=11,561$ & $\%$ & \\
\hline Lymphadenectomy (LN) & & & & & & & & & & & $<0.001$ \\
\hline$<15$ nodes & 12,918 & 44.8 & 3910 & 47.2 & 2644 & 46.6 & 1479 & 44.5 & 4885 & 42.3 & \\
\hline$\geq 15$ nodes & 14,086 & 48.8 & 3855 & 46.5 & 2643 & 46.6 & 1652 & 49.7 & 5936 & 51.4 & \\
\hline Unknown & 171 & 0.6 & 24 & 0.3 & 45 & 0.8 & 16 & 0.5 & 86 & 0.7 & \\
\hline LN Positive & & & & & & & & & & & $<0.001$ \\
\hline Negative & 15,727 & 54.5 & 5971 & 72.1 & 3566 & 62.8 & 1457 & 43.8 & 4733 & 40.9 & \\
\hline Positive & 11,224 & 38.9 & 1788 & 21.6 & 1717 & 30.2 & 1663 & 50.0 & 6056 & 52.4 & \\
\hline No LN examined & 1677 & 5.8 & 497 & 6.0 & 346 & 6.1 & 180 & 5.4 & 654 & 5.7 & \\
\hline Unknown & 224 & 0.8 & 30 & 0.4 & 49 & 0.9 & 27 & 0.8 & 118 & 1.0 & \\
\hline Pathological N stage & & & & & & & & & & & $<0.001$ \\
\hline No & 17,246 & 59.8 & 6461 & 78.0 & 3883 & 68.4 & 1608 & 48.3 & 5294 & 45.8 & \\
\hline $\mathrm{N}+$ & 11,462 & 39.7 & 1810 & 21.8 & 1758 & 31.0 & 1703 & 51.2 & 6191 & 53.6 & \\
\hline NX & 144 & 0.5 & 15 & 0.2 & 37 & 0.7 & 16 & 0.5 & 76 & 0.7 & \\
\hline Neoadjuvant Treatment & & & & & & & & & & & $<0.001$ \\
\hline Surgery first & 8187 & 28.4 & 5710 & 68.9 & 760 & 13.4 & 668 & 20.1 & 1049 & 9.1 & \\
\hline Chemotherapy alone & 2659 & 9.2 & 412 & 5.0 & 575 & 10.1 & 340 & 10.2 & 1332 & 11.5 & \\
\hline XRT alone & 340 & 1.2 & 51 & 0.6 & 72 & 1.3 & 53 & 1.6 & 164 & 1.4 & \\
\hline Chemoradiation & 17,666 & 61.2 & 2113 & 25.5 & 4271 & 75.2 & 2266 & 68.1 & 9016 & 78.0 & \\
\hline Adjuvant Treatment & & & & & & & & & & & $<0.001$ \\
\hline None & 24,127 & 83.6 & 7120 & 85.9 & 4840 & 85.2 & 2721 & 81.8 & 9446 & 81.7 & \\
\hline Chemotherapy alone & 2644 & 9.2 & 515 & 6.2 & 474 & 8.4 & 329 & 9.9 & 1326 & 11.5 & \\
\hline XRT alone & 443 & 1.5 & 56 & 0.7 & 108 & 1.9 & 48 & 1.4 & 231 & 2.0 & \\
\hline Chemoradiation & 1638 & 5.7 & 595 & 7.2 & 256 & 4.5 & 229 & 6.9 & 558 & 4.8 & \\
\hline Treatment Sequence & & & & & & & & & & & $<0.001$ \\
\hline Surgery only & 6079 & 21.1 & 4812 & 58.1 & 450 & 7.9 & 381 & 11.5 & 436 & 3.8 & \\
\hline Neoadjuvant & 18,048 & 62.6 & 2308 & 27.9 & 4390 & 77.3 & 2340 & 70.3 & 9010 & 77.9 & \\
\hline Adjuvant & 2108 & 7.3 & 898 & 10.8 & 310 & 5.5 & 287 & 8.6 & 613 & 5.3 & \\
\hline Neoadjuvant + adjuvant & 2617 & 9.1 & 268 & 3.2 & 528 & 9.3 & 319 & 9.6 & 1502 & 13.0 & \\
\hline
\end{tabular}

Abbreviations: LN, lymph node; T, tumor; TX, tumor not assessed; N, nodes; N+, node positive; NX, nodes not assessed; XRT, radiation therapy. 


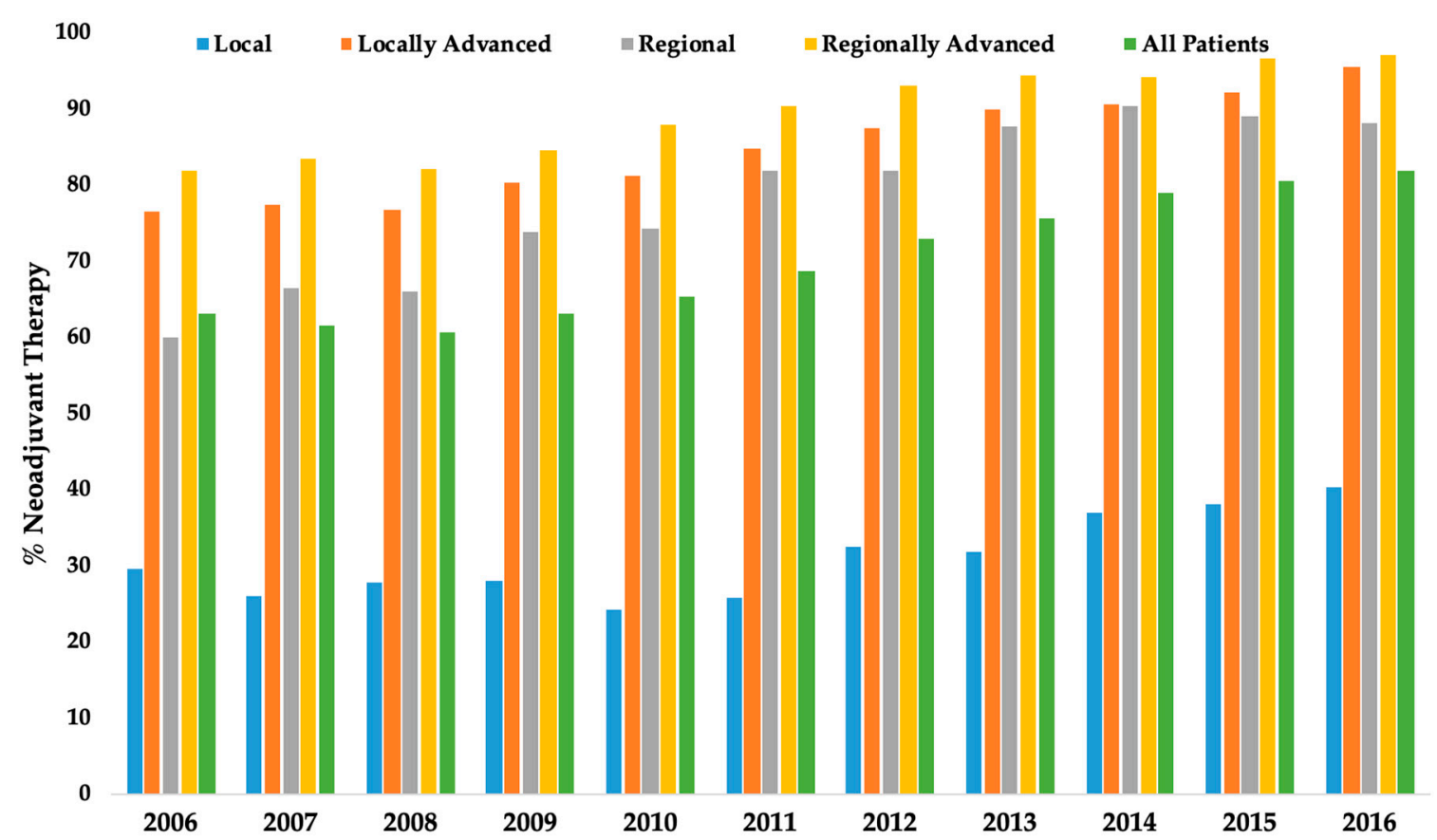

Figure 1. The use of neoadjuvant therapy (chemotherapy, radiation therapy, chemoradiation) by clinical stage from 2006 to 2016 for patients who underwent resection for gastroesophageal junction adenocarcinoma.

Table 3. Logistic regression analysis for factors associated with receiving NAT vs. surgery first.

\begin{tabular}{|c|c|c|c|c|}
\hline$n=28,852$ & OR & & CI & $p$ \\
\hline Esophagus vs. Stomach & 1.57 & 1.47 & 1.68 & \\
\hline $\begin{array}{l}\text { Gender } \\
\text { Male vs. Female }\end{array}$ & 1.33 & 1.22 & 1.46 & $<0.001$ \\
\hline $\begin{array}{c}\text { Age (ref. <50) } \\
50-59 \\
60-69 \\
70-79 \\
\geq 80\end{array}$ & $\begin{array}{l}1.02 \\
0.95 \\
0.66 \\
0.15\end{array}$ & $\begin{array}{l}0.90 \\
0.84 \\
0.57 \\
0.12\end{array}$ & $\begin{array}{l}1.17 \\
1.08 \\
0.76 \\
0.18\end{array}$ & $<0.001$ \\
\hline $\begin{array}{l}\text { Race (ref. Non White) } \\
\text { White } \\
\text { Unknown }\end{array}$ & $\begin{array}{l}1.27 \\
0.76\end{array}$ & $\begin{array}{l}1.13 \\
0.53\end{array}$ & $\begin{array}{l}1.44 \\
1.09\end{array}$ & $<0.001$ \\
\hline $\begin{array}{l}\text { Insurance (ref. Private) } \\
\text { Not Insured } \\
\text { Government plan } \\
\text { Unknown }\end{array}$ & $\begin{array}{l}1.09 \\
0.92 \\
0.72\end{array}$ & $\begin{array}{l}0.84 \\
0.85 \\
0.56\end{array}$ & $\begin{array}{l}1.42 \\
0.99 \\
0.93\end{array}$ & 0.014 \\
\hline $\begin{array}{l}\text { Facility Type } \\
\text { Academic vs. Community }\end{array}$ & 0.90 & 0.84 & 0.97 & 0.004 \\
\hline $\begin{array}{l}\text { Comorbidity score (ref. } \geq 2 \text { ) } \\
0 \\
1\end{array}$ & $\begin{array}{l}1.43 \\
1.18\end{array}$ & $\begin{array}{l}1.27 \\
1.04\end{array}$ & $\begin{array}{l}1.61 \\
1.34\end{array}$ & $<0.001$ \\
\hline $\begin{array}{l}\text { Clinical stage (ref. T3-4N+) } \\
\text { T1-2N0 } \\
\text { T3-4N0 } \\
\text { T1-2N+ }\end{array}$ & $\begin{array}{l}0.04 \\
0.69 \\
0.40\end{array}$ & $\begin{array}{l}0.04 \\
0.63 \\
0.35\end{array}$ & $\begin{array}{l}0.05 \\
0.77 \\
0.44\end{array}$ & $<0.001$ \\
\hline
\end{tabular}

Abbreviations: NAT, neoadjuvant therapy; OR, odds ratio; ref., reference; CI, confidence Interval. 
The median overall survival for GEJA patients undergoing resection was 3.3 years. Median overall survival was significantly greater in patients who received NAT than in patients who underwent upfront surgery in the LA (3.3 years vs. 2.1 years, $p<0.001$ ), R (3.4 years vs. 2.4 years, $p<0.001$ ), and RA (2.6 years vs. 1.7 years, $p<0.001$ ) groups (Figure 2). In the Cox regression model, the interaction terms between the use of NAT and clinical stage were significant (L: HR $=1.53, \mathrm{CI}=1.43-1.65$; LA: HR $=0.75$, $\mathrm{CI}=0.68-0.83 ; \mathrm{R}: \mathrm{HR}=0.86, \mathrm{CI}=0.77-0.96 ; \mathrm{RA}: \mathrm{HR}=0.73, \mathrm{CI}=0.68-0.79, p<0.001$ ), indicating the survival impact of NAT is different by clinical stage. Therefore, Cox regression models were fit for each clinical stage category with only pretreatment factors. The factors associated with worse OS are listed in Table A2 by clinical stage. Use of NAT was associated with improved OS in LA (HR $=0.75$, $\mathrm{CI}=0.68-0.83, p<0.001), \mathrm{R}(\mathrm{HR}=0.87, \mathrm{CI}=0.77-0.98, p=0.017)$, and $\mathrm{RA}(\mathrm{HR}=0.70, \mathrm{CI}=0.65-0.75$, $p<0.001)$, but not in $\mathrm{L}(\mathrm{HR}=1.56, \mathrm{CI}=1.45-1.68, p<0.001)$ patients after adjustments with other factors (Figure 2).

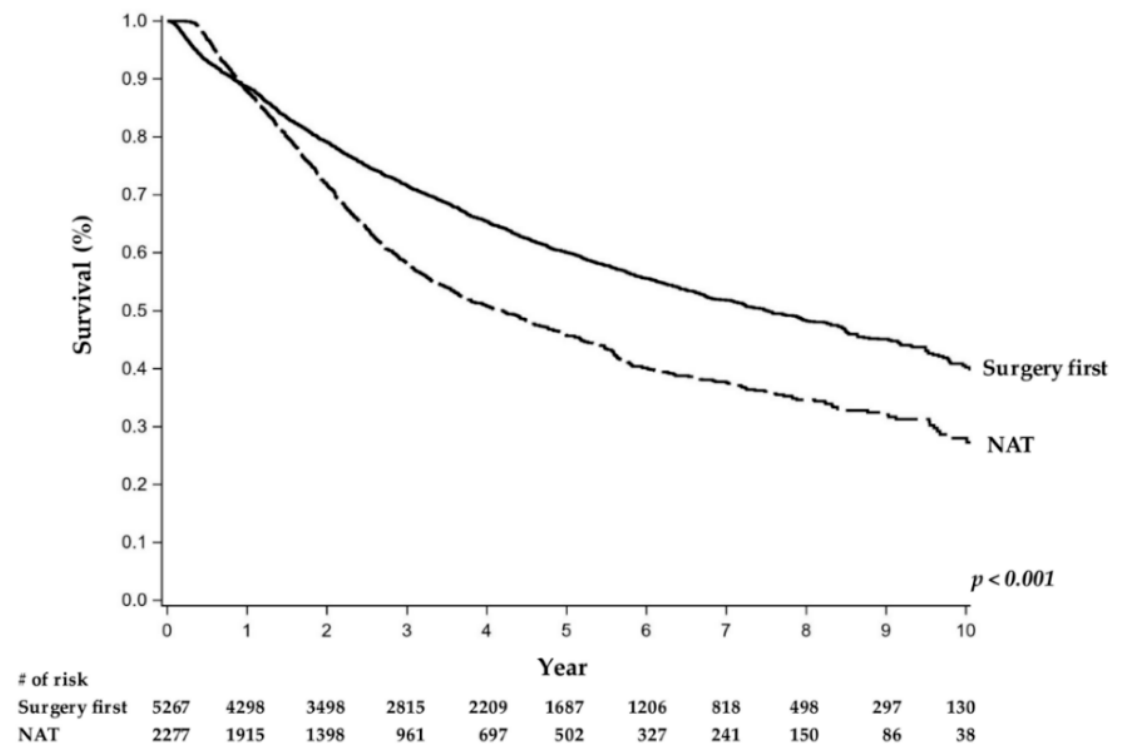

(A)

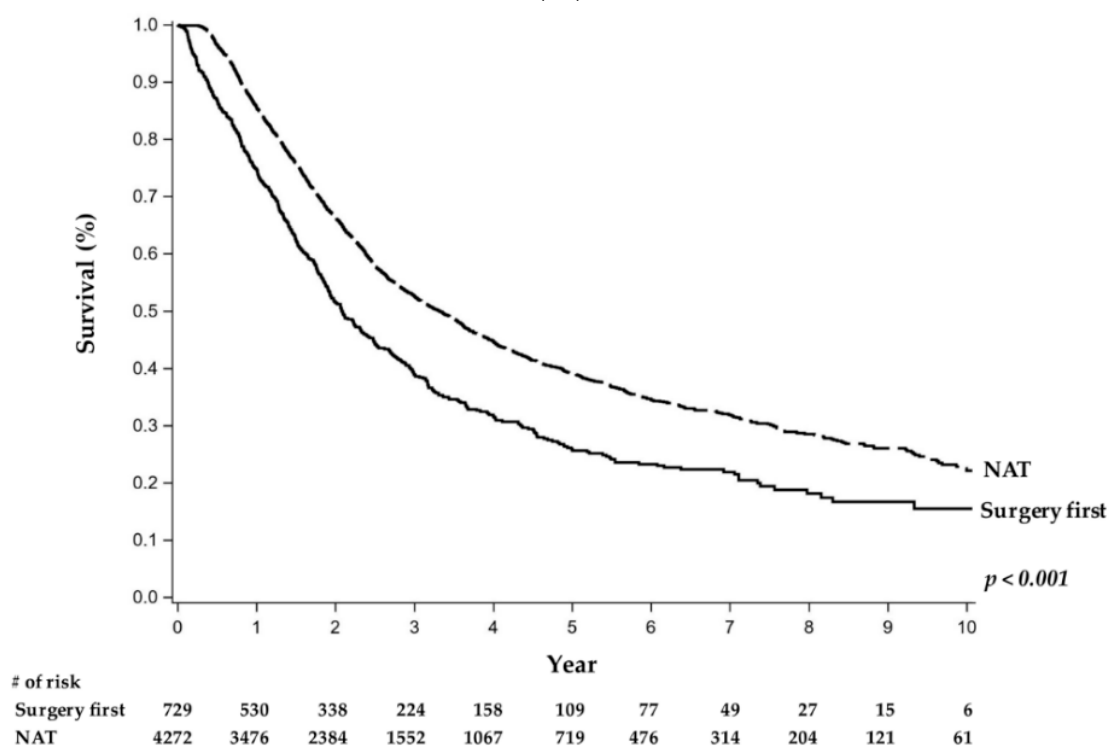

(B)

Figure 2. Cont. 


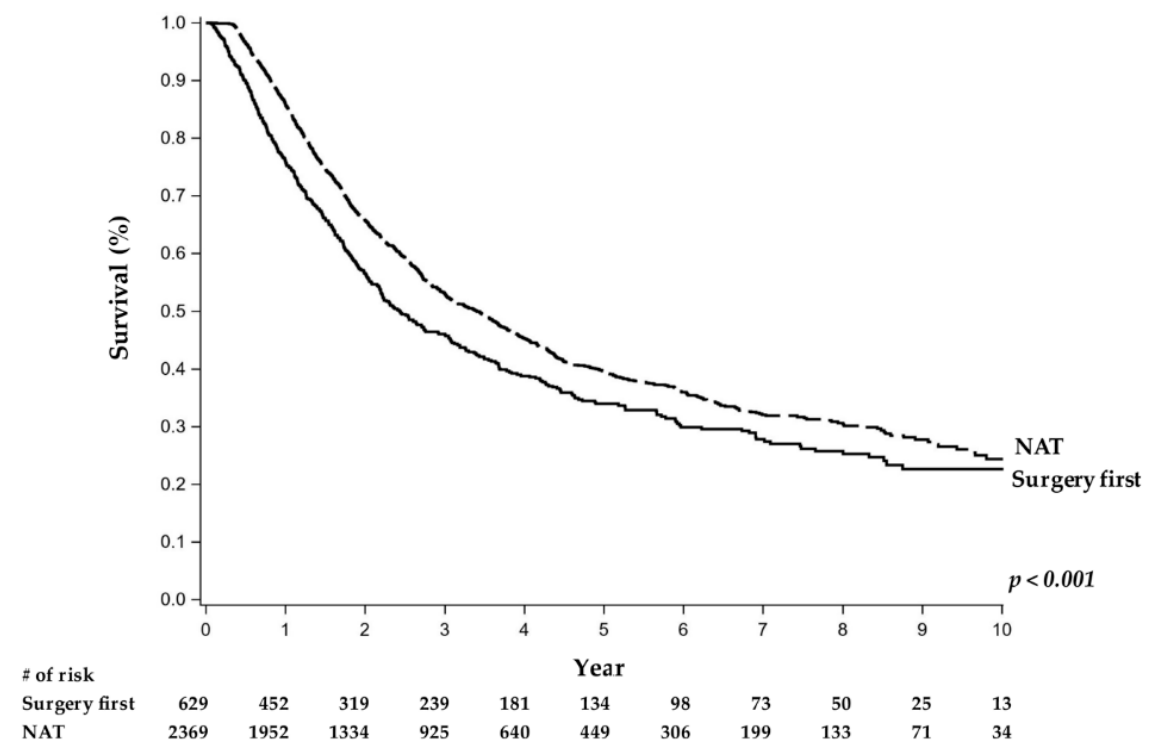

(C)

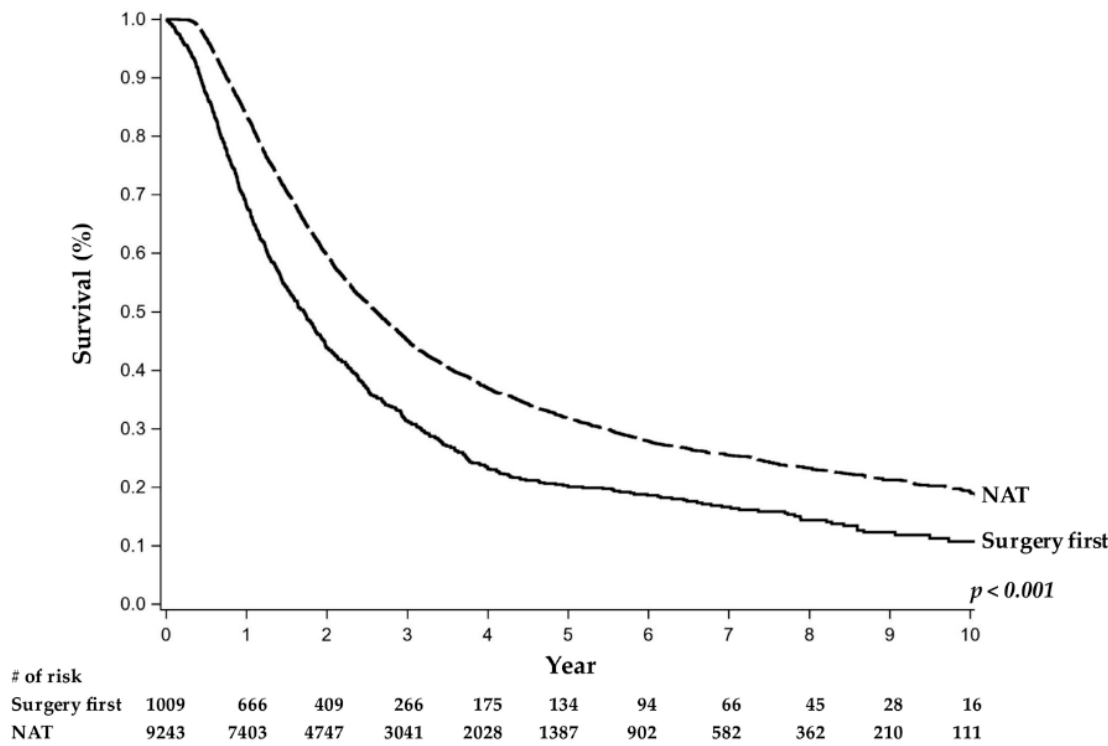

(D)

Figure 2. Overall survival analysis comparing patients with gastroesophageal junction adenocarcinoma who received upfront surgery to those who first received neoadjuvant therapy across all clinical stages: (A) early localized (T1-2N0), (B) locally advanced (T3-4N0), (C) regional (T1-2N+), and (D) regionally advanced (T3-4N+). Abbreviations: NAT, neoadjuvant therapy. \#, number.

\section{Discussion}

This study represents the largest and most modern analysis of current treatment patterns for resected GEJA in the United States. It identified a large volume of patients undergoing resection for GEJA and showed that the majority of patients with clinical stage LA, R, and RA disease received NAT. Despite the lack of strong evidence showing survival benefit of preoperative chemoradiation therapy over chemotherapy alone for GEJA, most patients who underwent NAT received chemoradiation therapy. A remarkable increase in the use of NAT was observed annually over the decade of this study's period. As previously reported, nodal upstaging was more frequent in patients with clinical stage L disease, in particular when upfront surgery was performed without NAT. 
Based on data from the SEER cancer registry, a remarkable increase in the incidence of esophageal adenocarcinoma at the lower esophageal location was observed from the 1970s to early 2000s, and a substantial proportion of this increase was due to an increase in the incidence of GEJA $[13,14]$. Although this rise has slowed from a 10\% annual increase before 1999 to a $1.6 \%$ decline in subsequent years, GEJA continues to be a major public health problem in the United States. The authors of these studies speculated that exposure to the initiating factors (reflux disease or increased body mass index) for esophageal adenocarcinoma have plateaued in exerting maximal potential effect, and that the premalignant transformation of Barrett's esophagus to cancer could have slowed with screening and surveillance. Our analysis of NCDB showed a persistent high volume of GEJA.

Neoadjuvant therapy for surgically resectable gastric, esophageal, and junctional cancers is well supported by level I evidence $[6-8,15,16]$. As a result, the use of neoadjuvant therapy has rapidly increased for esophageal and gastric cancers over the past two decades [17-19]. Our study supplements this information with a more granular analysis specifically for GEJA, in which the treatment strategy is particularly controversial. This study demonstrated that the uptake of NAT has continued to increase in recent years. Despite the lack of high-level evidence supporting the superiority of chemoradiation therapy over chemotherapy alone for GEJA [20,21], a very high proportion of the patients who underwent NAT received chemoradiation therapy in the United States. This could be explained by previous U.S. population studies showing that $82 \%$ of gastric cardia cancers are treated by esophagectomy rather than gastrectomy. Therefore, surgeons (likely thoracic surgeons rather than abdominal surgeons) who perform esophagectomy are thought to be more familiar with CROSS trial chemoradiation over MAGIC trial chemotherapy for gastrectomies [22]. The potential benefits of neoadjuvant chemoradiation therapy over chemotherapy alone for GEJA include higher rates of pathological complete response and margin-negative (R0) resection [23], which may result in improved local control [12]. In surgical resection of advanced GEJA, it is often challenging to achieve R0 margins because of the tumor's narrow and deep anatomical location; therefore, the local effect of chemoradiation therapy may be more beneficial for GEJ tumors than non-GEJ esophageal or gastric cancers. The survival benefit of neoadjuvant chemoradiation therapy vs. chemotherapy alone is being investigated in the international phase III Trial of Preoperative Therapy for Gastric and Esophagogastric Junction Adenocarcinoma (TOPGEAR) trial [24]. The TOPGEAR trial randomly assigned patients with gastric or GEJ adenocarcinomas to receive perioperative chemotherapy (epirubicin/cisplatin/5-fluorouracil) with or without preoperative chemoradiation therapy (45 Gy in 25 fractions with concurrent 5-fluorouracil). Similarly, the Dutch phase II CRITICS-II trial compared preoperative chemotherapy and chemoradiation therapy in gastric and GEJ adenocarcinomas [25]. Although these trials were not specifically designed for GEJA, the results may have implications for the future clinical treatment of GEJA.

There are some limitations of this study. First, the retrospective study design is inherently subject to selection bias. However, this large nationwide cohort provides useful information about the increasing use of neoadjuvant therapy for resected GEJA. To our knowledge, this study is the first nationwide analysis investigating trends in the treatment approach specifically for GEJA. Second, the NCDB lacks granularity, which makes analysis of GEJA challenging, as the tumors are coded in several ways nationally from an esophagus or gastric perspective (lower third of the esophagus vs. abdominal esophagus vs. gastric cardia). We created a dataset that is suggestive of GEJA origin but likely included tumors without involvement of GEJ. And although contributing centers to the NCDB include various types of hospitals that range from community hospitals to academic cancer centers, generalizability of the study results to non-NCDB hospitals may be limited. Next, the survival analyses conducted in this study, particularly in assessment of survival impact of the use of NAT, were severely limited due to selection bias and immortal time bias associated with the use and duration of NAT. Although the models we fit in this study suggested possible survival benefit of NAT in GEJA that support the increased use of NAT as shown in this study, the optimal regimen (particularly comparing chemoradiation therapy vs. chemotherapy only) should be investigated by prospective trials, and results of the above-mentioned 
trials are awaited. Lastly, this study did not analyze specific methods of resection (esophagectomy or gastrectomy) or surgical approach (open vs. minimally invasive or transthoracic vs. transhiatal), because the purpose of this study was to examine current treatment patterns and sequences of GEJA and not methods of surgical approach. Further, several studies have emphasized the importance of preoperative therapy rather than a surgical approach in oncologic outcomes, specifically overall survival, for patients with this disease [26,27].

\section{Conclusions}

In this study analyzing esophageal and gastric datasets from the NCDB, lower-third esophageal and cardia gastric adenocarcinomas continued to be the main proportion of esophageal and gastric cancers in the United States. The use of NAT for GEJA has steadily increased over the past decade, except for early localized disease, for which upfront surgery is the most common approach. Most patients who underwent NAT received chemoradiation therapy, despite the lack of existing evidence supporting any survival benefit of chemoradiation therapy over chemotherapy alone. Results of ongoing prospective randomized controlled trials may impact future clinical practice for GEJA in the United States.

Author Contributions: Conceptualization, B.J.K. and N.I.; Data curation B.J.K. and Y.-J.C.; Formal analysis, B.J.K., Y.-J.C. and N.I.; Investigation, B.J.K. and N.I.; Methodology, B.J.K., Y.-J.C. and N.I.; Supervision, N.I.; Validation, B.J.K. and Y.-J.C.; Visualization, B.J.K., Y.-J.C. and N.I.; Writing-original draft, B.J.K., Y.-J.C. and N.I.; Writing-review \& editing, B.J.K., Y.-J.C., P.D., B.D.M., M.A.B., J.A.A., J.S.E., W.L.H., C.-W.D.T., B.D.B., P.F.M. and N.I. All authors have read and agreed to the published version of the manuscript.

Funding: This research received no external funding.

Acknowledgments: Editorial support was provided by Bryan Tutt in Editing Services, Research Medical Library.

Conflicts of Interest: The authors declare no conflict of interest.

\section{Appendix A}

Table A1. Overall number of esophageal and gastric cases combined, overall number of Gastroesophageal Junction Adenocarcinoma (GEJA) cases, and rate of resection.

\begin{tabular}{cccccc}
\hline & Overall & \multicolumn{2}{c}{ Overall GEJA } & \multicolumn{2}{c}{ Resected GEJA } \\
\cline { 2 - 6 } & $\boldsymbol{n}$ & $\boldsymbol{n}$ & $\mathbf{\%}$ & $\boldsymbol{n}$ & $\mathbf{\%}$ \\
\hline 2006 & 17,118 & 8838 & 51.6 & 3337 & 37.8 \\
2007 & 17,596 & 8856 & 50.3 & 3269 & 36.9 \\
2008 & 17,960 & 9227 & 51.4 & 3279 & 35.5 \\
2009 & 18,718 & 9721 & 51.9 & 3380 & 34.8 \\
2010 & 18,794 & 9986 & 53.1 & 3336 & 33.4 \\
2011 & 19,471 & 10,393 & 53.4 & 3521 & 33.9 \\
2012 & 20,063 & 10,720 & 53.4 & 3490 & 32.6 \\
2013 & 20,756 & 11,299 & 54.4 & 3726 & 33.0 \\
2014 & 21,156 & 11,597 & 54.8 & 3737 & 32.2 \\
2015 & 21,437 & 11,995 & 56.0 & 3787 & 31.6 \\
2016 & 21,212 & 11,918 & 56.2 & 3649 & 30.6 \\
\hline
\end{tabular}

Abbreviations: $n$, number; GEJA, gastroesophageal junction adenocarcinoma. Overall GEJA $\%$ : proportion of patients with Gastroesophageal Junction (GEJ) tumors among overall patients for each year. Resected GEJA\%: proportion of resected GEJA among overall GEJ. 
Table A2. Cox regression model for overall survival by clinical stage of gastroesophageal adenocarcinoma.

\begin{tabular}{|c|c|c|c|c|}
\hline \multicolumn{5}{|l|}{ Early Localized: T1-2N0 ( $n=7544)$} \\
\hline & HR & \multicolumn{2}{|c|}{$95 \% \mathrm{CI}$} & \multirow{2}{*}{$\begin{array}{c}p \\
0.014\end{array}$} \\
\hline Stomach vs. Esophagus & 1.09 & 1.02 & 1.17 & \\
\hline Age (ref. <50) & & & & $<0.001$ \\
\hline $50-59$ & 1.07 & 0.90 & 1.27 & \\
\hline $60-69$ & 1.20 & 1.02 & 1.41 & \\
\hline $70-79$ & 1.55 & 1.31 & 1.84 & \\
\hline$\geq 80$ & 2.63 & 2.16 & 3.21 & \\
\hline Race (ref. Non White) & & & & 0.048 \\
\hline White & 1.18 & 1.02 & 1.37 & \\
\hline Unknown & 0.88 & 0.54 & 1.43 & \\
\hline Insurance (ref. private) & & & & $<0.001$ \\
\hline Non insured & 1.96 & 1.53 & 2.50 & \\
\hline Government's plan & 1.29 & 1.18 & 1.41 & \\
\hline Unknown & 1.26 & 0.96 & 1.64 & \\
\hline Facility Type & & & & $<0.001$ \\
\hline Academic vs. Community & 0.80 & 0.75 & 0.86 & \\
\hline Comorbidity score (ref. 0) & & & & 0.003 \\
\hline 1 & 1.09 & 1.01 & 1.19 & \\
\hline$\geq 2$ & 1.21 & 1.07 & 1.37 & \\
\hline Education (ref. $\geq 29 \%$ ) & & & & 0.001 \\
\hline$<29 \%$ & 0.93 & 0.83 & 1.04 & \\
\hline Zip code income (ref. $\geq \$ 46,000$ ) & & & & $<0.001$ \\
\hline$<\$ 46,000$ & 1.17 & 1.08 & 1.26 & \\
\hline Neoadjuvant treatment & & & & $<0.001$ \\
\hline NAT vs. Surgery only & 1.56 & 1.45 & 1.68 & \\
\hline \multicolumn{5}{|l|}{ Locally Advanced: T3-4N0 ( $n=5003)$} \\
\hline & HR & \multicolumn{2}{|c|}{$95 \%$ CI } & $p$ \\
\hline Gender & & & & 0.003 \\
\hline Female vs. Male & 0.85 & 0.76 & 0.94 & \\
\hline Age (ref. <50) & & & & $<0.001$ \\
\hline $50-59$ & 1.13 & 0.97 & 1.32 & \\
\hline $60-69$ & 1.15 & 0.99 & 1.34 & \\
\hline $70-79$ & 1.46 & 1.24 & 1.71 & \\
\hline$\geq 80$ & 2.03 & 1.63 & 2.54 & \\
\hline Insurance (ref. private) & & & & 0.013 \\
\hline Non insured & 1.09 & 0.82 & 1.46 & \\
\hline Government's plan & 1.16 & 1.06 & 1.27 & \\
\hline Unknown & 1.23 & 0.91 & 1.66 & \\
\hline Facility Type & & & & 0.031 \\
\hline Academic vs. Community program & 0.92 & 0.85 & 0.99 & \\
\hline Comorbidity score (ref. 0) & & & & 0.002 \\
\hline 1 & 1.15 & 1.06 & 1.26 & \\
\hline$\geq 2$ & 1.18 & 1.02 & 1.36 & \\
\hline Neoadjuvant treatment & & & & $<0.001$ \\
\hline NAT vs. Surgery only & 0.75 & 0.68 & 0.83 & \\
\hline
\end{tabular}


Table A2. Cont.

\begin{tabular}{|c|c|c|c|c|}
\hline \multicolumn{5}{|l|}{ Regional: $\mathrm{T} 1-2 \mathrm{~N}+(n=2998)$} \\
\hline & HR & \multicolumn{2}{|c|}{$95 \%$ CI } & \multirow{3}{*}{$\begin{array}{c}p \\
0.026\end{array}$} \\
\hline & & & & \\
\hline Stomach vs. Esophagus & 1.12 & 1.01 & 1.23 & \\
\hline Gender & & & & 0.044 \\
\hline Female vs. Male & 0.86 & 0.74 & 1.00 & \\
\hline Age (ref. <50) & & & & $<0.001$ \\
\hline $50-59$ & 0.93 & 0.77 & 1.12 & \\
\hline $60-69$ & 1.04 & 0.87 & 1.24 & \\
\hline $70-79$ & 1.28 & 1.06 & 1.54 & \\
\hline$\geq 80$ & 2.01 & 1.52 & 2.66 & \\
\hline Comorbidity score (ref. 0) & & & & $<0.001$ \\
\hline 1 & 1.25 & 1.12 & 1.40 & \\
\hline$\geq 2$ & 1.10 & 0.90 & 1.34 & \\
\hline Zip code income (ref. $\geq \$ 46,000)$ & & & & 0.009 \\
\hline$<\$ 46,000$ & 1.16 & 1.06 & 1.29 & \\
\hline unknown & 1.14 & 0.85 & 1.52 & \\
\hline Neoadjuvant treatment & & & & 0.017 \\
\hline NAT vs. Surgery only & 0.87 & 0.77 & 0.98 & \\
\hline \multicolumn{5}{|l|}{ Regionally Advanced: T3-4N+ (10,255) } \\
\hline & HR & \multicolumn{2}{|c|}{$95 \%$ CI } & $p$ \\
\hline Gender & & & & 0.001 \\
\hline Female vs. Male & 0.89 & 0.82 & 0.95 & \\
\hline Age (ref. <50) & & & & $<0.001$ \\
\hline $50-59$ & 1.05 & 0.96 & 1.15 & \\
\hline $60-69$ & 1.08 & 0.99 & 1.18 & \\
\hline $70-79$ & 1.24 & 1.12 & 1.37 & \\
\hline$\geq 80$ & 1.58 & 1.34 & 1.86 & \\
\hline Race (ref. Non White) & & & & 0.002 \\
\hline White & 1.21 & 1.09 & 1.34 & \\
\hline Unknown & 1.10 & 0.80 & 1.52 & \\
\hline Insurance (ref. private) & & & & $<0.001$ \\
\hline Non insured & 1.17 & 0.98 & 1.40 & \\
\hline Government's plan & 1.15 & 1.08 & 1.22 & \\
\hline Unknown & 1.04 & 0.84 & 1.29 & \\
\hline Facility Type & & & & $<0.001$ \\
\hline Academic vs. Community program & 0.91 & 0.86 & 0.96 & \\
\hline Comorbidity score (ref. 0) & & & & $<0.001$ \\
\hline 1 & 1.10 & 1.04 & 1.17 & \\
\hline$\geq 2$ & 1.24 & 1.12 & 1.37 & \\
\hline Zipcode income (ref. $\geq \$ 46,000$ ) & & & & $<0.001$ \\
\hline$<\$ 46,000$ & 1.14 & 1.08 & 1.20 & \\
\hline unknown & 0.98 & 0.84 & 1.15 & \\
\hline Neoadjuvant treatment & & & & $<0.001$ \\
\hline NAT vs. Surgery only & 0.70 & 0.65 & 0.75 & \\
\hline
\end{tabular}

Abbreviations: HR, hazard ratio; NAT, neoadjuvant therapy; ref., reference; CI, confidence Interval; T, tumor; $\mathrm{N}$, nodes. 


\section{References}

1. Buas, M.F.; Vaughan, T.L. Epidemiology and risk factors for gastroesophageal junction tumors: Understanding the rising incidence of this disease. Semin. Radiat. Oncol. 2013, 23, 3-9. [CrossRef] [PubMed]

2. Siewert, J.R.; Holscher, A.H.; Becker, K.; Gossner, W. Cardia cancer: Attempt at a therapeutically relevant classification. Chirurg 1987, 58, 25-32. [PubMed]

3. Siewert, J.R.; Stein, H.J.; Sendler, A.; Fink, U. Surgical resection for cancer of the cardia. Semin. Surg. Oncol. 1999, 17, 125-131. [CrossRef]

4. Macdonald, J.S.; Smalley, S.R.; Benedetti, J.; Hundahl, S.A.; Estes, N.C.; Stemmermann, G.N.; Haller, D.G.; Ajani, J.A.; Gunderson, L.L.; Jessup, J.M.; et al. Chemoradiotherapy after surgery compared with surgery alone for adenocarcinoma of the stomach or gastroesophageal junction. N. Engl. J. Med. 2001, 345, 725-730. [CrossRef] [PubMed]

5. Sakuramoto, S.; Sasako, M.; Yamaguchi, T.; Kinoshita, T.; Fujii, M.; Nashimoto, A.; Furukawa, H.; Nakajima, T.; Ohashi, Y.; Imamura, H.; et al. Adjuvant chemotherapy for gastric cancer with S-1, an oral fluoropyrimidine. N. Engl. J. Med. 2007, 357, 1810-1820. [CrossRef] [PubMed]

6. Cunningham, D.; Allum, W.H.; Stenning, S.P.; Thompson, J.N.; Van de Velde, C.J.; Nicolson, M.; Scarffe, J.H.; Lofts, F.J.; Falk, S.J.; Iveson, T.J.; et al. Perioperative chemotherapy versus surgery alone for resectable gastroesophageal cancer. N. Engl. J. Med. 2006, 355, 11-20. [CrossRef] [PubMed]

7. Ychou, M.; Boige, V.; Pignon, J.P.; Conroy, T.; Bouche, O.; Lebreton, G.; Ducourtieux, M.; Bedenne, L.; Fabre, J.M.; Saint-Aubert, B.; et al. Perioperative chemotherapy compared with surgery alone for resectable gastroesophageal adenocarcinoma: An FNCLCC and FFCD multicenter phase III trial. J. Clin. Oncol. 2011, 29, 1715-1721. [CrossRef]

8. Van Hagen, P.; Hulshof, M.C.; van Lanschot, J.J.; Steyerberg, E.W.; van Berge Henegouwen, M.I.; Wijnhoven, B.P.; Richel, D.J.; Nieuwenhuijzen, G.A.; Hospers, G.A.; Bonenkamp, J.J.; et al. Preoperative chemoradiotherapy for esophageal or junctional cancer. N. Engl. J. Med. 2012, 366, 2074-2084. [CrossRef]

9. Tepper, J.; Krasna, M.J.; Niedzwiecki, D.; Hollis, D.; Reed, C.E.; Goldberg, R.; Kiel, K.; Willett, C.; Sugarbaker, D.; Mayer, R. Phase III trial of trimodality therapy with cisplatin, fluorouracil, radiotherapy, and surgery compared with surgery alone for esophageal cancer: CALGB 9781. J. Clin. Oncol. 2008, 26, 1086-1092. [CrossRef]

10. National Comprehensive Cancer Network. NCCN Clinical Practice Guidelines in Oncology Esophageal and Esophagogastric Junction Cancers. Available online: https://www.nccn.org/professionals/physician_gls/pdf/ esophageal.pdf (accessed on 20 June 2020).

11. Stahl, M.; Walz, M.K.; Stuschke, M.; Lehmann, N.; Meyer, H.J.; Riera-Knorrenschild, J.; Langer, P.; Engenhart-Cabillic, R.; Bitzer, M.; Konigsrainer, A.; et al. Phase III comparison of preoperative chemotherapy compared with chemoradiotherapy in patients with locally advanced adenocarcinoma of the esophagogastric junction. J. Clin. Oncol. 2009, 27, 851-856. [CrossRef]

12. Stahl, M.; Walz, M.K.; Riera-Knorrenschild, J.; Stuschke, M.; Sandermann, A.; Bitzer, M.; Wilke, H.; Budach, W. Preoperative chemotherapy versus chemoradiotherapy in locally advanced adenocarcinomas of the oesophagogastric junction (POET): Long-term results of a controlled randomised trial. Eur. J. Cancer 2017, 81, 183-190. [CrossRef] [PubMed]

13. Pohl, H.; Sirovich, B.; Welch, H.G. Esophageal adenocarcinoma incidence: Are we reaching the peak? Cancer Epidemiol. Biomark. Prev. 2010, 19, 1468-1470. [CrossRef] [PubMed]

14. Pohl, H.; Welch, H.G. The role of overdiagnosis and reclassification in the marked increase of esophageal adenocarcinoma incidence. J. Natl. Cancer Inst. 2005, 97, 142-146. [CrossRef] [PubMed]

15. Schuhmacher, C.; Gretschel, S.; Lordick, F.; Reichardt, P.; Hohenberger, W.; Eisenberger, C.F.; Haag, C.; Mauer, M.E.; Hasan, B.; Welch, J.; et al. Neoadjuvant chemotherapy compared with surgery alone for locally advanced cancer of the stomach and cardia: European Organisation for Research and Treatment of Cancer randomized trial 40954. J. Clin. Oncol. 2010, 28, 5210-5218. [CrossRef]

16. Xu, A.M.; Huang, L.; Liu, W.; Gao, S.; Han, W.X.; Wei, Z.J. Neoadjuvant chemotherapy followed by surgery versus surgery alone for gastric carcinoma: Systematic review and meta-analysis of randomized controlled trials. PLoS ONE 2014, 9, e86941. [CrossRef] 
17. Greenleaf, E.K.; Hollenbeak, C.S.; Wong, J. Trends in the use and impact of neoadjuvant chemotherapy on perioperative outcomes for resected gastric cancer: Evidence from the American College of Surgeons National Cancer Database. Surgery 2016, 159, 1099-1112. [CrossRef]

18. Aversa, J.G.; Diggs, L.P.; Hagerty, B.L.; Dominguez, D.A.; Wiemken, T.; Luu, C.; Hernandez, J.M. Trends of Clinician Adherence to Evidence-Based Recommendations for Multidisciplinary Oncology Care for Patients With Esophageal Cancer. JAMA Oncol. 2020. [CrossRef]

19. Ikoma, N.; Cormier, J.N.; Feig, B.; Du, X.L.; Yamal, J.M.; Hofstetter, W.; Das, P.; Ajani, J.A.; Roland, C.L.; Fournier, K.; et al. Racial disparities in preoperative chemotherapy use in gastric cancer patients in the United States: Analysis of the National Cancer Data Base, 2006-2014. Cancer 2018, 124, 998-1007. [CrossRef]

20. Al-Sukhni, E.; Gabriel, E.; Attwood, K.; Kukar, M.; Nurkin, S.J.; Hochwald, S.N. No Survival Difference with Neoadjuvant Chemoradiotherapy Compared with Chemotherapy in Resectable Esophageal and Gastroesophageal Junction Adenocarcinoma: Results from the National Cancer Data Base. J. Am. Coll. Surg. 2016, 223, 784-792.e1. [CrossRef]

21. Munch, S.; Habermehl, D.; Agha, A.; Belka, C.; Combs, S.E.; Eckel, R.; Friess, H.; Gerbes, A.; Nussler, N.C.; Schepp, W.; et al. Perioperative chemotherapy vs. neoadjuvant chemoradiation in gastroesophageal junction adenocarcinoma: A population-based evaluation of the Munich Cancer Registry. Strahlenther. Onkol. 2018, 194, 125-135. [CrossRef]

22. Martin, J.T.; Mahan, A.; Zwischenberger, J.B.; McGrath, P.C.; Tzeng, C.W. Should gastric cardia cancers be treated with esophagectomy or total gastrectomy? A comprehensive analysis of 4,996 NSQIP/SEER patients. J. Am. Coll. Surg. 2015, 220, 510-520. [CrossRef]

23. Zafar, S.N.; Blum, M.; Chiang, Y.J.; Ajani, J.A.; Estrella, J.S.; Das, P.; Minsky, B.D.; Hofstetter, W.L.; Mansfield, P.; Badgwell, B.D.; et al. Preoperative Chemoradiation versus Chemotherapy in Gastroesophageal Junction Adenocarcinoma. Ann. Thorac. Surg. 2020. [CrossRef] [PubMed]

24. Leong, T.; Smithers, B.M.; Haustermans, K.; Michael, M.; Gebski, V.; Miller, D.; Zalcberg, J.; Boussioutas, A.; Findlay, M.; O'Connell, R.L.; et al. TOPGEAR: A Randomized, Phase III Trial of Perioperative ECF Chemotherapy with or Without Preoperative Chemoradiation for Resectable Gastric Cancer: Interim Results from an International, Intergroup Trial of the AGITG, TROG, EORTC and CCTG. Ann. Surg. Oncol. 2017, 24, 2252-2258. [CrossRef] [PubMed]

25. Slagter, A.E.; Jansen, E.P.M.; van Laarhoven, H.W.M.; van Sandick, J.W.; van Grieken, N.C.T.; Sikorska, K.; Cats, A.; Muller-Timmermans, P.; Hulshof, M.; Boot, H.; et al. CRITICS-II: A multicentre randomised phase II trial of neo-adjuvant chemotherapy followed by surgery versus neo-adjuvant chemotherapy and subsequent chemoradiotherapy followed by surgery versus neo-adjuvant chemoradiotherapy followed by surgery in resectable gastric cancer. BMC Cancer 2018, 18, 877. [CrossRef]

26. Kneuertz, P.J.; Hofstetter, W.L.; Chiang, Y.J.; Das, P.; Blum, M.; Elimova, E.; Mansfield, P.; Ajani, J.; Badgwell, B. Long-Term Survival in Patients with Gastroesophageal Junction Cancer Treated with Preoperative Therapy: Do Thoracic and Abdominal Approaches Differ? Ann. Surg. Oncol. 2016, 23, 626-632. [CrossRef] [PubMed]

27. Koeter, M.; Parry, K.; Verhoeven, R.H.; Luyer, M.D.; Ruurda, J.P.; van Hillegersberg, R.; Lemmens, V.E.; Nieuwenhuijzen, G.A. Perioperative Treatment, Not Surgical Approach, Influences Overall Survival in Patients with Gastroesophageal Junction Tumors: A Nationwide, Population-Based Study in The Netherlands. Ann. Surg. Oncol. 2016, 23, 1632-1638. [CrossRef]

Publisher's Note: MDPI stays neutral with regard to jurisdictional claims in published maps and institutional affiliations.

(C) 2020 by the authors. Licensee MDPI, Basel, Switzerland. This article is an open access article distributed under the terms and conditions of the Creative Commons Attribution (CC BY) license (http://creativecommons.org/licenses/by/4.0/). 